01

\title{
Сдвиги частоты магнитного резонанса спин-поляризованных атомов цезия в смеси Cs-Rb
}

\author{
(C) B.A. Картошкин \\ Физико-технический институт им. А.Ф.Иофффе РАН, \\ 194021 Санкт-Петербург, Россия \\ e-mail: victor.kart@mail.ioffe.ru
}

Поступила в редакцию 28.02.2018 г.

Рассмотрено взаимодействие спин-поляризованных атомов цезия с атомами цезия и рубидия в условиях оптической ориентации атомов. На основании данных по комплексным сечениям спинового обмена для систем Cs-Cs и Cs- $\mathrm{Rb}$ проводится расчет сдвига частоты магнитного резонанса атомов цезия в смеси $\mathrm{Cs}-\mathrm{Rb}$, а также сравнение расчетных значений сдвига частоты с экспериментальными данными.

DOI: $10.21883 /$ OS.2018.07.46259.62-18

\section{Введение}

Как известно, спин-обменные столкновения между щелочными атомами играют важную роль в приборах квантовой электроники - квантовых магнитометрах, стандартах частоты и времени. Наряду с переносом поляризации между сталкивающимися частицами спинобменные столкновения приводят к сдвигу частоты магнитного резонанса сталкивающихся атомов. К настоящему времени вопросы влияния спинового обмена на процессы переноса поляризации и связанные с ними вопросы релаксации достаточно хорошо изучены как экспериментально $[1,2]$, так и теоретически (в том числе исследованы спин-обменные процессы с участием ядерных парамагнетиков) [3,4]. В то же время сдвиги частоты магнитного резонанса, связанные с этим процессом, изучены существенно меньше. Особенно это касается экспериментальных измерений сдвигов и расчетов температурных зависимостей сечений сдвигов частоты. В [5,6] впервые были измерены сдвиги частоты магнитного резонанса для атомов Cs [5] при спинобменных столкновениях с атомами $\mathrm{Rb}$ и атомов $\mathrm{Rb}$ при столкновении с атомами Cs [6]. В [7] была предпринята попытка расчета сечений сдвигов частоты магнитного резонанса для гомоядерных пар щелочных атомов. Однако проведенный расчет сечений и последующая оценка точности квантовых стандартов частоты проводились с использованием оценочных выражений для потенциалов взаимодействия. В [7] синглетный и триплетный потенциалы взаимодействия оценивались исходя из потенциалов ионизации сталкивающихся атомов. Вместе с тем, в [8] были получены потенциалы взаимодействия для триплетного и синглетного состояний систем Rb-Rb, Cs-Cs и Cs-Rb. Приведенные потенциалы взаимодействия позволили рассчитать интересующие сечения сдвигов частоты. Однако дальнейшие экспериментальные исследования с помощью методов фурьеспектроскопии систем $\mathrm{Cs}_{2}$ (для синглетного $\mathrm{X}^{1} \Sigma^{+}$[9] и триплетного $\mathrm{a}^{3} \Sigma^{+}[10]$ термов) и $\mathrm{CsRb}$ (для син- глетного $\mathrm{X}^{1} \Sigma^{+}$и триплетного $\mathrm{a}^{3} \Sigma^{+}[10]$ термов [11]) дали возможность получить более точную информацию по потенциалам взаимодействия исследуемых систем. В дальнейшем на основании полученных в этих работах потенциалов взаимодействия были рассчитаны комплексные сечения спинового обмена для $\mathrm{Cs}_{2}$ [12] и $\mathrm{CsRb}$ [13]. Полученные результаты позволили рассчитать сдвиги частоты магнитного резонанса для атомов Cs в смеси $\mathrm{Cs}-\mathrm{Rb}$ и провести их сравнение c экспериментальными данными.

\section{Комплексные сечения спинового обмена при столкновении щелочных атомов}

Расчет сдвигов частоты магнитного резонанса проводился в рамках теории, изложенной в [14]. Следует отметить, что полученные в работе выражения, связывающие сдвиги частоты магнитного резонанса, обусловленные спин-обменными столкновениями, найдены с учетом не только спин-обменного процесса, но также с учетом влияния диффузии к стенкам камеры поглощения и столкновений с атомными частицами буферного газа. Кроме того, полученные выражения учитывают сложную систему атомных уровней щелочных атомов.

В настоящей работе впервые приводится расчет сдвигов частоты магнитного резонанса атомов Cs в смеси щелочных атомов $\mathrm{Cs}-\mathrm{Rb}$ в атмосфере буферного газа $\left(\mathrm{N}_{2}\right)$ и их сравнение с величинами сдвигов, измеренными в экспериментах по оптической ориентации атомов.

При столкновении двух атомов щелочных металлов, находящихся в основном состоянии, происходит хорошо известный процесс спинового обмена, т. е. обмен валентными электронами между сталкивающимися атомами. Такие столкновения приводят к переносу поляризации между сталкивающимися атомными частицами и поэтому могут быть использованы для передачи поляризации от одного объекта столкновения другому. Подобного 


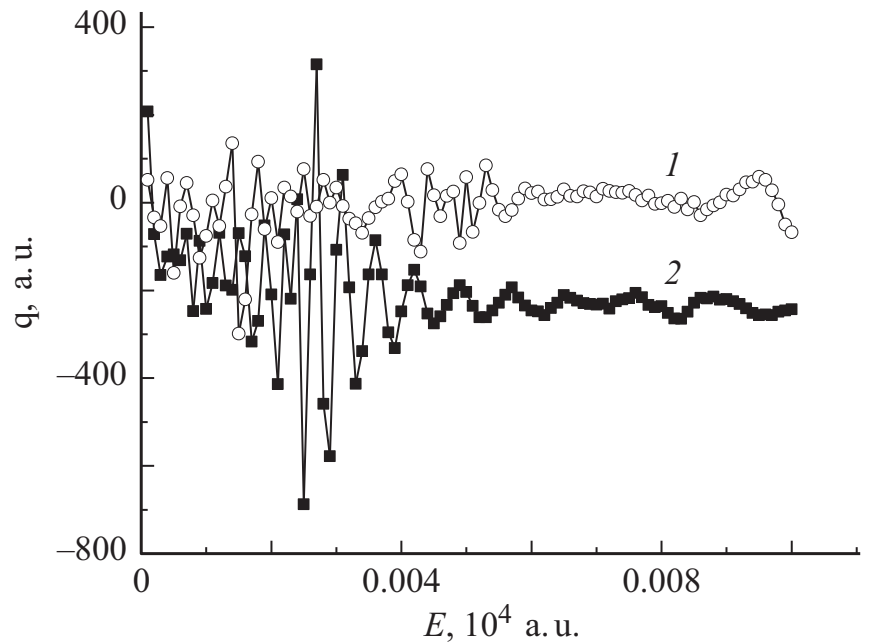

Рис. 1. Зависимость мнимой части комплексного сечения спинового обмена от энергии сталкивающихся частиц (в атомной системе единиц): 1 - сечения сдвигов для $\mathrm{Cs}-\mathrm{Cs}, 2-$ сечения сдвигов для $\mathrm{Cs}-\mathrm{Rb}$.

рода непрямая поляризация атомных частиц хорошо известна и широко применяется в работах по оптической ориентации атомов.

Процесс спинового обмена может быть описан с помощью комплексного сечения $q_{01}^{A B}=\overline{q_{01}^{A B}}+i \overline{\overline{q_{01}^{A B}}}$. При этом действительная часть сечения $\overline{q_{01}^{A B}}$ определяет перенос ориентации при столкновении, в то время как мнимая часть сечения $\overline{\overline{q_{01}^{A B}}}-$ сдвиг частоты магнитного резонанса.

Комплексное сечение спинового обмена может быть представлено в виде

$$
q_{01}^{A B}=\frac{\pi}{k_{A B}^{2}} \sum_{l=0}^{\infty}(2 l+1)\left[1-T_{0}^{A B}(l) T_{1}^{A B}(l)^{*}\right] .
$$

Здесь $k_{A B}-$ волновой вектор, символ $*$ указывает на комплексное сопряжение, $l$ - орбитальное квантовое число, $T_{S}^{A B}(l)$ - матрица рассеяния, явный вид которой определяется стандартным образом:

$$
T_{S}^{A B}(l)=\exp \left(2 i \delta_{S}^{A B}(l)\right),
$$

где $\delta_{S}^{A B}(l)$ - фаза рассеяния на терме с полным спином $S$.

Используя потенциалы взаимодействия димеров $\mathrm{Cs}_{2}$ и $\mathrm{CsRb}$, проведем расчет сдвигов для атомов цезия и сравним расчетные и экспериментальные значения. На рис. 1 приведены рассчитанные ранее $[12,13]$ зависимости мнимых частей комплексного сечения взаимодействия от энергии столкновения частиц для систем $\mathrm{Cs}-\mathrm{Cs}$ и Cs-Rb. В дальнейшем данные по сечениям используются для расчета сдвигов частоты магнитного резонанса.

\section{Сдвиги частоты магнитного резонанса при столкновении поляризованных щелочных атомов}

В [14] приводятся теоретическое рассмотрение спинового обмена с участием щелочных атомов в условиях эксперимента по оптической ориентации атомов, а также выражения, описывающие сдвиги частоты магнитного резонанса этих атомов.

Учет влияния в кинетических уравнениях вклада, связанного с деполяризацией щелочных атомов на стенке камеры поглощения и в объеме при столкновении с атомами буферного газа, необходим, когда скорость спинового обмена $\overline{\gamma_{A B}}$ сравнима со скоростью релаксации, обусловленной диффузией $\gamma_{D}$ и столкновениями с атомами буферных газов $\gamma_{A C}$ [14]. Действительно, скорость диффузии определяется как

$$
\gamma_{D}=\frac{D}{\lambda_{D}^{2}}
$$

где $D$ - коэффициент диффузии атомов Cs в буферном газе (в данном случае в молекулярном азоте), $\lambda_{D}-$ диффузионная длина, определяемая из линейных размеров камеры поглощения (в работе [5] использовались камеры поглощения диаметром $4 \mathrm{~cm}$ и длиной $6 \mathrm{~cm}$, что дает $1 / \lambda_{D}^{2}=1.71 \mathrm{~cm}^{-2}$ при давлении буферного газа 100 Torr). Вместе с тем наличие буферного газа в камере поглощения приводит также к разрушению поляризации щелочного атома при столкновениях, однако, как следует из [15] (для атомов рубидия), совокупное влияние диффузии и деполяризации при столкновении с буферным газом приводит к скоростям деполяризации, существенно более низким, чем скорости спинового обмена $\overline{\gamma_{A B}}$ и $\overline{\gamma_{A A}}$. Более подробно ситуация была рассмотрена в [16]. Таким образом, при расчете сдвигов частоты магнитного резонанса можно воспользоваться выражениями, которые получены без учета диффузии и деполяризации на буферном газе, что существенно упрощает расчеты.

Учет только спин-обменных столкновений приводит к следующим выражениям для сдвигов:

$$
\begin{aligned}
& \delta^{(1)} \omega(+)=-\frac{P_{z}(B)}{2\left(2 I_{A}+1\right)}\left[\overline{\overline{\gamma_{A B}}}-\overline{\overline{\gamma_{A A}}} B_{-}\left(\frac{2 I_{A}-1}{2 I_{A}+1}\right)^{1 / 2}\right], \\
& \delta^{(1)} \omega(-)=-\frac{P_{z}(B)}{2\left(2 I_{A}+1\right)}\left[\overline{\overline{\gamma_{A B}}}+\overline{\overline{\gamma_{A A}}} B_{+}\left(\frac{2 I_{A}+3}{2 I_{A}+1}\right)^{1 / 2}\right] .
\end{aligned}
$$

В соответствии с обозначениями, принятыми в [14], здесь $\delta^{(1)} \omega(+)$ - сдвиг частоты магнитного резонанса для сверхтонкого состояния $F=S+I$ (где $S$ - электронный спин, в случае щелочных атомов он равен $1 / 2$, а $I$ - ядерный спин щелочного атома), $\delta^{(1)} \omega(-)-$ сдвиг частоты магнитного резонанса для сверхтонкого состояния $F=S-I, P_{z}(B)-$ поляризация частицы $B$, $I_{A}$ - ядерный спин частицы $A, \overline{\overline{\gamma_{A B}}}$ и $\overline{\overline{\gamma_{A A}}}$ - мнимые 
части комплексной скорости спинового обмена $\gamma$, которая может быть выражена через комплексное сечение спинового обмена (1). Индексы $A A$ и $A B$ соответствуют столкновениям между одинаковыми или разными щелочными атомами:

$$
\gamma_{A B}=\left\langle v_{A B}\right\rangle N_{B} \sigma_{A B},
$$

где

$$
\sigma_{A B}(T)=\frac{\left\langle q_{01}^{A B}(E) v\right\rangle}{\langle v\rangle}=\int_{0}^{\infty} q_{01}^{A B}(E) E \exp (-E / k T) d E .
$$

Здесь $E$ - кинетическая энергия столкновения атомов, $k$ - постоянная Больцмана, $T-$ температура, $q_{01}^{A B}(E)$ - сечение, определяемое выражением (1), $N_{B}$ концентрация частиц $B, v_{A B}$ - относительная скорость сталкивающихся частиц. При столкновении одинаковых атомов индекс $B$ заменяется на $A$.

В [5] в экспериментах по оптической ориентации атомов были измерены сдвиги частоты магнитного резонанса атомов цезия $-{ }^{133} \mathrm{Cs}\left(I_{A}=7 / 2\right)$ для двух сверхтонких состояний $F_{A}=I_{A}+1 / 2=4$ и $F_{A}=I_{A}-1 / 2=3$. Камера поглощения, содержавшая смесь изотопа ${ }^{87} \mathrm{Rb}\left(I_{A}=3 / 2\right)$ и ${ }^{133} \mathrm{Cs}$ в равных весовых количествах, заполненная буферным газом - азотом при давлении 100 Torr, помещалась в термостат. Изменение концентрации щелочных атомов в камере поглощения осуществлялось путем изменения температуры термостата от 20 до $80^{\circ} \mathrm{C}$. Резонансным излучением линии рубидия осуществлялась оптическая ориентация атомов Rb. Степень поляризации щелочных атомов в подобного рода экспериментах обычно не превышает нескольких десятков процентов. Далее возбуждался магнитный резонанс на зеемановской частоте атомов цезия для каждого из сверхтонких состояний, что приводило к изменению поглощения света накачки атомами рубидия за счет процесса спинового обмена между Cs и Rb. Изменение поляризации света накачки с $(+)$ на $(-)$ позволяло регистрировать удвоенную частоту сдвига частоты магнитного резонанса. На рис. 2 из [5] приведены зависимости модулей удвоенного сдвига частоты магнитного резонанса $\left(\left|2 \delta f_{ \pm}\right|\right)$от температуры в камере поглощения ( \pm относится к $F=4$ и $F=3$ соответственно). Концентрация атомов щелочных металлов определялась в соответствии с таблицами, приведенными в [17]. Поскольку в эксперименте использовалась смесь щелочных атомов, то для перехода от температуры камеры поглощения к концентрации в ней щелочных атомов необходимо воспользоваться законом Рауля для давления насыщенного пара над расплавом смеси металлов.

Для расчета сдвигов частоты магнитного резонанса, определяемых выражениями (4), (5), необходимо знание мнимой части комплексного сечения спинового обмена (1). Температурная зависимость этой величины

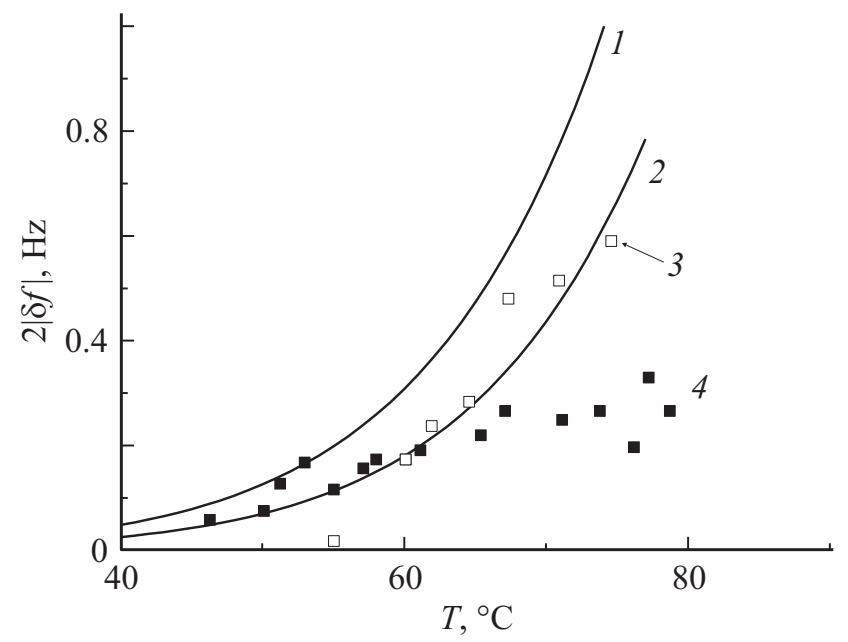

Рис. 2. Зависимости удвоенного сдвига частоты магнитного резонанса атомов Cs в смеси $\mathrm{Cs}-\mathrm{Rb}$ от температуры: 1 расчетная величина $2|\delta f|$ для состояния $F=3,2-$ расчетная величина $2|\delta f|$ для состояния $F=4,3-$ экспериментальная величина $2|\delta f|$ для состояния $F=3$ по данным [5], $4-$ экспериментальная величина $2|\delta f|$ для состояния $F=4$ по данным [5].

$\left(\sigma_{A A}\right.$ и $\left.\sigma_{A B}\right)$ для пар атомов $\mathrm{Cs}-\mathrm{Cs}$ и $\mathrm{Rb}-\mathrm{Cs}$ была рассчитана ранее $[12,13]$.

Таким образом, оказывается возможным рассчитать сдвиги частоты магнитного резонанса на основании соотношений (4) и (5), используя рассчитанные величины сечений. На рис. 2 приводятся результаты такого расчета для электронной поляризации атома $B P_{z}(B)=0.3$ (в нашем случае это атом Rb).

\section{Заключение}

Как видно из рисунка, при поляризации 30\% абсолютные значения расчетных и экспериментальных значений сдвигов различаются для сверхтонких состояний $F=4$ и $F=3$. Ранее в экспериментах по измерению сдвигов частоты магнитного резонанса атомов $\mathrm{Rb}$ при столкновении с атомами Cs сдвиги для двух сверхтонких состояний рубидия совпадали. В экспериментах с атомами Cs, как видно из рисунка, также наблюдается различие сдвигов для сверхтонких состояний, что соответствует поведению расчетных зависимостей сдвигов для двух сверхтонких состояний.

Основным источником погрешности как при обработке эксперимента, так и при переходе в расчетах от сечений к сдвигам является неопределенность концентраций щелочных атомов, особенно в случае смеси металлов. Кроме того, на точность сравнения эксперимента и теории влияет имеющаяся неопределенность степени поляризации атомов. При этом надо отметить, что в эксперименте проявилось предсказанное теорией различие сдвигов для двух сверхтонких состояний Cs. 


\section{Список литературы}

[1] Happer W. // Rev. Mod. Phys. 1972. V. 4. N 2. P. 169-249.

[2] Vanier J., Audoin C. The quantum physics of atomic frequency standards. Bristol: Adam Hilger, 1989.

[3] Appelt A., Ben Amar Baranga et al. // Phys. Rev. A. 1998. V. 58. N 2. P. $1412-1439$.

[4] Окуневич А.И. Оптическая ориентация атомов и молекул. Л., 1987. С. $65-76$.

[5] Дмитриев С.П., Доватор Н.А. // ЖТФ. 1997. Т. 67. В. 2. C. 115.

[6] Дмитриев С.П., Доватор Н.А. // ЖТФ. 2007. Т. 77. В. 7. C. 120.

[7] Micalizio S., Godone A., Levi F., Vanier J. // Phys. Rev. A. 2006. V. 73. N 3. P. 033414.

[8] Картошкин В.А. // Опт. и спектр. 1995. Т. 79. № 1. С. 26.

[9] Aviot C., Dulieu // J. Chem. Phys. 2002. V. 117. P. 5155.

[10] Xie F., Sovkov V.B. et al. // J. Chem. Phys. 2009. V. 130. P. 051102

[11] Docencko O., Tamanis R., Ferber R. et al. // Phys. Rev. A. 2011. V. 83. P. 052519.

[12] Дмитриев С.П., Доватор Н.А., Картошкин В.А. // ЖТФ. 2015. T. 60. В. 6. С. 826.

[13] Картошкин В.А. // Опт. и спектр. 2016. Т. 121. № 3. С. 327.

[14] Окуневич А.И. // Опт. и спектр. 1995. Т. 79. № 5. С. 718.

[15] Wagshul M.E., Chupp T.E. // Phys. Rev. A. 1994. V. 49. N 5. P. 3854.

[16] Картошкин В.А. // Опт. и спектр. 2010. Т. 108. № 6. С. 866.

[17] Несмеянов А.Н. Давление паров химических элементов. М., 1961.396 c. 